\title{
Case Report Methylmalonic Acidemia with Novel MUT Gene Mutations
}

\author{
Inusha Panigrahi, Savita Bhunwal, Harish Varma, and Simranjeet Singh \\ Department of Pediatrics, Advanced Pediatric Centre, PGIMER, Chandigarh, India \\ Correspondence should be addressed to Inusha Panigrahi; inupan@yahoo.com
}

Received 6 June 2017; Revised 5 August 2017; Accepted 14 September 2017; Published 12 October 2017

Academic Editor: Yoshiyuki Ban

\begin{abstract}
Copyright (C) 2017 Inusha Panigrahi et al. This is an open access article distributed under the Creative Commons Attribution License, which permits unrestricted use, distribution, and reproduction in any medium, provided the original work is properly cited.

A 5-year-old boy presented with recurrent episodes of fever, feeding problems, lethargy, from the age of 11 months, and poor weight gain. He was admitted and evaluated for metabolic causes and diagnosed as having methylmalonic acidemia (MMA). He was treated with vit B12 and carnitine supplements and has been on follow-up for the last 3 years. Mutation analysis by next generation sequencing (NGS), supplemented with Sanger sequencing, revealed two novel variants in the MUT gene responsible for MMA in exon 5 and exon 3, respectively. Recently he developed dystonic movements including orofacial dyskinesia. With advent of NGS, judicious use of NGS with Sanger sequencing can help identify causative possibly pathogenic mutations.
\end{abstract}

\section{Case Presentation}

The child presented for the first time at the age of 11 months, with complaints of fever, vomiting, poor feeding, and lethargy. On examination he had pallor and tachypnea and was drowsy. Further evaluation was suggestive of high anion-gap metabolic acidosis with ketonuria (urine ketones$3+$ ) with normal electrolytes, blood sugar ( $94 \mathrm{mg} / \mathrm{dl})$, vitamin B12, and homocysteine. Plasma ammonia was 118 units, and plasma lactate was 2.9 units. TMS was normal but urine GCMS revealed elevated 3-OH propionic acid [12.39 retention time (RT)] as well as elevated methyl malonic acid [16.92 RT, Suppl Figure 1, in Supplementary Material available online at https://doi.org/10.1155/2017/8984951]. Since then this child was on low protein diet, carnitine, biotin, thiamine, and vitamin B12 injections. Child was thereafter admitted on multiple occasions (7 times) with acute decompensation and managed as per protocol. Mutational analysis was sent for MMA which showed single heterozygous missense variant c.976 A>G (p.Arg326Gly) in exon 5 of $M U T$ gene (genomic coordinates: $\operatorname{chr}$ 6: 49421405) as a variant of uncertain significance. Chromosomal microarray analysis done did not reveal any major deletion or duplication which could disrupt the gene. Since exon 3 and exon 6 were not adequately covered by NGS, further evaluation by Sanger sequencing for targeted exons was done and a 2nd mutation in exon 3 c.753 G>A (p.=) was identified. The variants were found to be damaging on SIFT database score (Suppl data). They were also predicted to be deleterious on Polyphen-2 and Mutation Taster and not found in the ExAC database. MRI brain done at the age of 4 years was showing multifocal cystic encephalomalacic changes with surrounding gliosis in deep white matter predominantly in frontoparietal regions (Figure 1).

In latest admission child was found to have fresh neurological findings in the form of perioral tremors, generalised hypertonia, and generalised dystonia with clonus with exaggerated deep tendon reflexes. He was treated with intravenous dextrose and sodium bicarbonate and was continued on carnitine and injection of vitamin B12. Plasma ammonia was 18 units and lactate level was 4.9 units. MRI brain was repeated and revealed bilateral basal ganglia hyperintensities suggestive of metabolic stroke. After the subsidence of acute crisis he was discharged on carnitine, injection of vitamin B12, and trihexyphenidyl. Parents were counseled regarding prognosis and for prenatal diagnosis next pregnancy.

\section{Discussion}

MMA presents with lethargy, acidosis, hypoglycemia/ hyperglycemia, ketosis, and recurrent episodes. MMA due to MUT gene mutations usually led to severe phenotype, and around $35-40 \%$ of cases are due to new mutations $[1,2]$. There can be missense or nonmutations, deletions, insertions, 


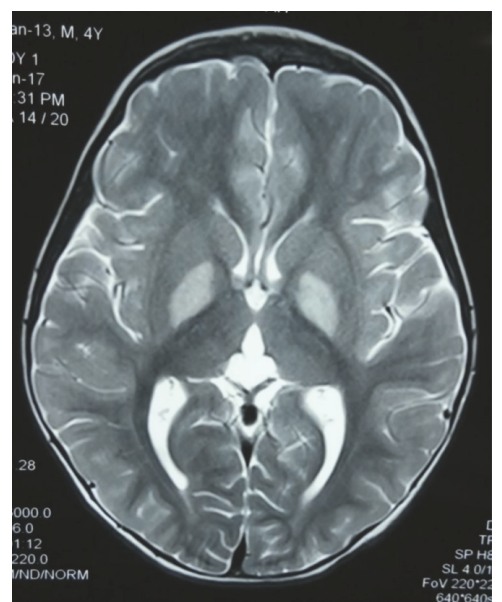

FIGURE 1: The MRI brain in the child with MUT-related MMA showing predominant frontoparietal abnormalities in form of encephalomalacia and gliosis.

and so on leading to clinical phenotype. The advent of NGS technology has enabled better characterization of mutations in several populations. However, Sanger sequencing remains useful adjunct in molecular testing of these cases. Sometimes in NGS, due to incomplete coverage of the exons, Sanger sequencing is required to find mutations, if there is strong clinical suspicion. By careful use of both techniques, we could find the two variants responsible for the clinical condition. In a Saudi study on 60 patients of MMA, nonsense, missense, and frameshift mutations were detected across the MUT gene [3]. Another study in 43 Chinese patients identified 8 recurrent mutations and 10 novel mutations [4]. A previous Indian study in 15 patients of clinically diagnosed MMA identified one novel exon 12 mutation in MUT gene with predicted pathogenicity. Here, we identified two novel variants, one in exon 3 and another in exon 5 of the MUT gene. Both were labelled as variants of unknown significance (VUS). The exon 3 variant is a synonymous variant, and a different nucleotide change c.753 G>C (p.Lys251Asn) has been reported earlier in ClinVar. Some synonymous variants can also affect the splicing or protein function and lead to clinical phenotypes. The identified exon 5 variant is new, but another close variant c.977 G>A (p.Arg326Lys) has been reported in ClinVar. The variants were found to be deleterious on bioinformatic analysis and were not found in ExAC database. Both variants identified in present case possibly explain the phenotype of MMA in the child.

MUT-related MMA has poor prognosis in most cases. Specialised diet and supplements may not improve outcomes, even if diagnosed early. Early recognition and appropriate treatment of acute crises are necessary. Metabolic stroke can sometimes occur in the absence of acute metabolic decompensation, so meticulous neurological examination at each visit is useful. The options for therapy include early liver transplantation [5] and possibly gene therapy in the future. Genetic counseling and prenatal diagnosis help these families in making reproductive decisions.

\section{Conflicts of Interest}

The authors declare that they have no conflicts of interest.

\section{Acknowledgments}

The authors would like to acknowledge Dhiti Omics Technologies Pvt Ltd for help in mutation analysis.

\section{References}

[1] K. Splinter, A.-K. Niemi, R. Cox et al., "Impaired health-related quality of life in children and families affected by methylmalonic acidemia," Journal of Genetic Counseling, vol. 25, no. 5, pp. 936-944, 2016.

[2] A. R. R. Devi and S. M. Naushad, "Targeted exome sequencing for the identification of complementation groups in methylmalonic aciduria: a south Indian experience," Clinical Biochemistry, vol. 50, no. 1-2, pp. 68-72, 2017.

[3] F. Imtiaz, B. M. Al-Mubarak, A. Al-Mostafa et al., "Spectrum of mutations in 60 saudi patients with mut methylmalonic acidemia," JIMD Reports, vol. 29, pp. 39-46, 2016.

[4] L.-S. Han, Z. Huang, F. Han et al., "Clinical features and MUT gene mutation spectrum in Chinese patients with isolated methylmalonic acidemia: identification of ten novel allelic variants," World Journal of Pediatrics, vol. 11, no. 4, pp. 358-365, 2015.

[5] M. Spada, P. L. Calvo, A. Brunati et al., "Liver transplantation in severe methylmalonic acidemia: the sooner, the better," Journal of Pediatrics, vol. 167, no. 5, p. 1173, 2015. 


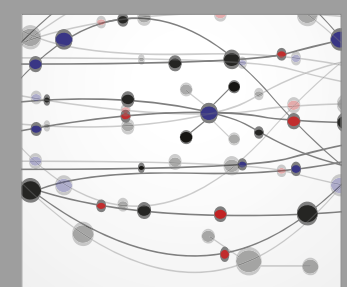

The Scientific World Journal
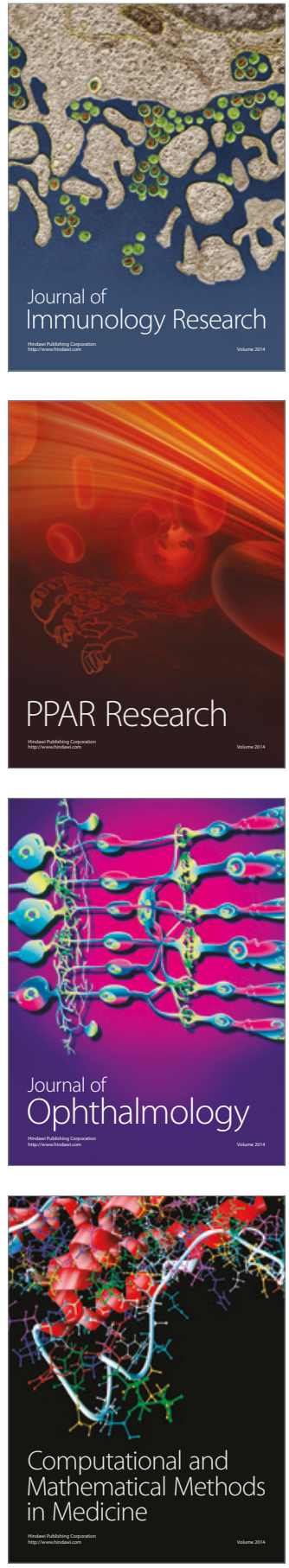

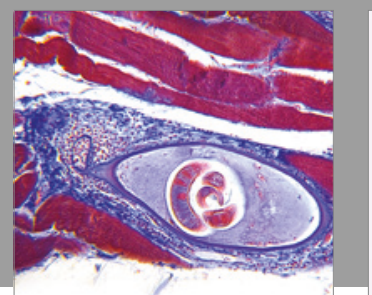

Gastroenterology Research and Practice
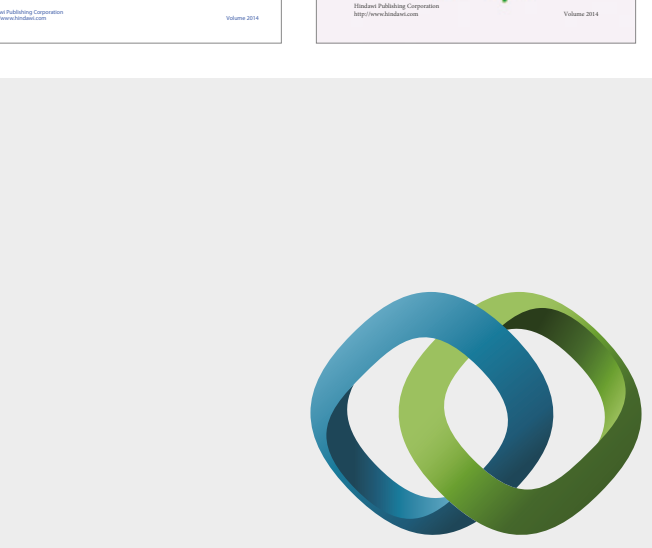

\section{Hindawi}

Submit your manuscripts at

https://www.hindawi.com
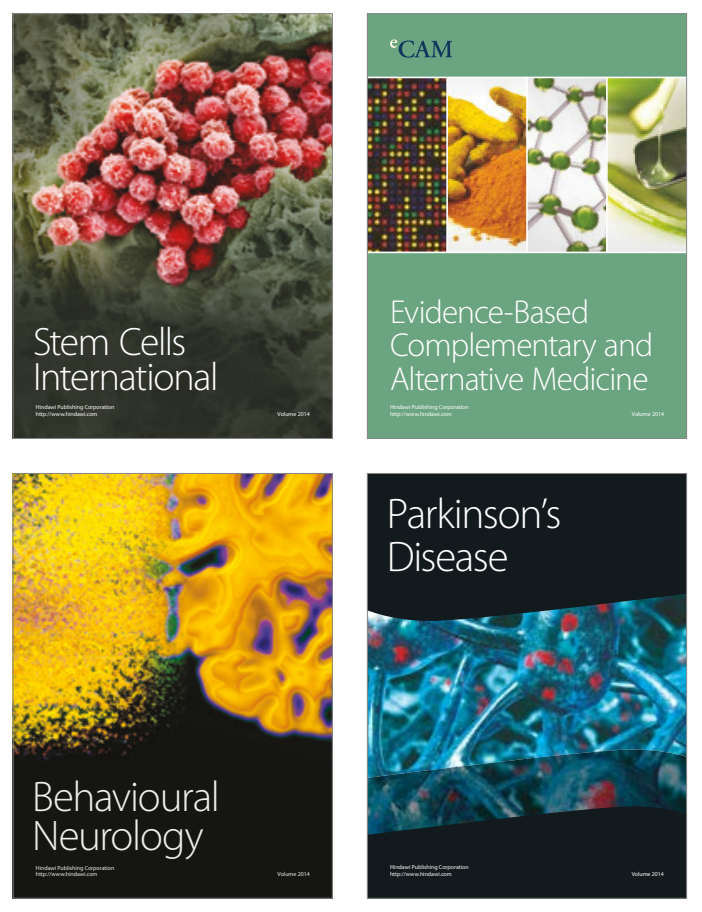
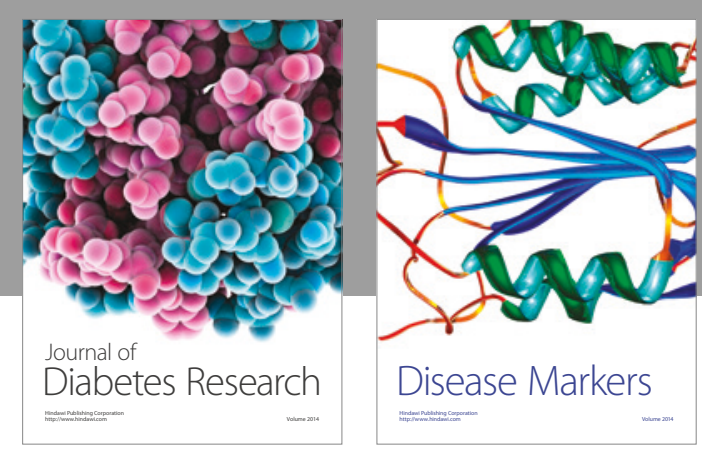

Disease Markers
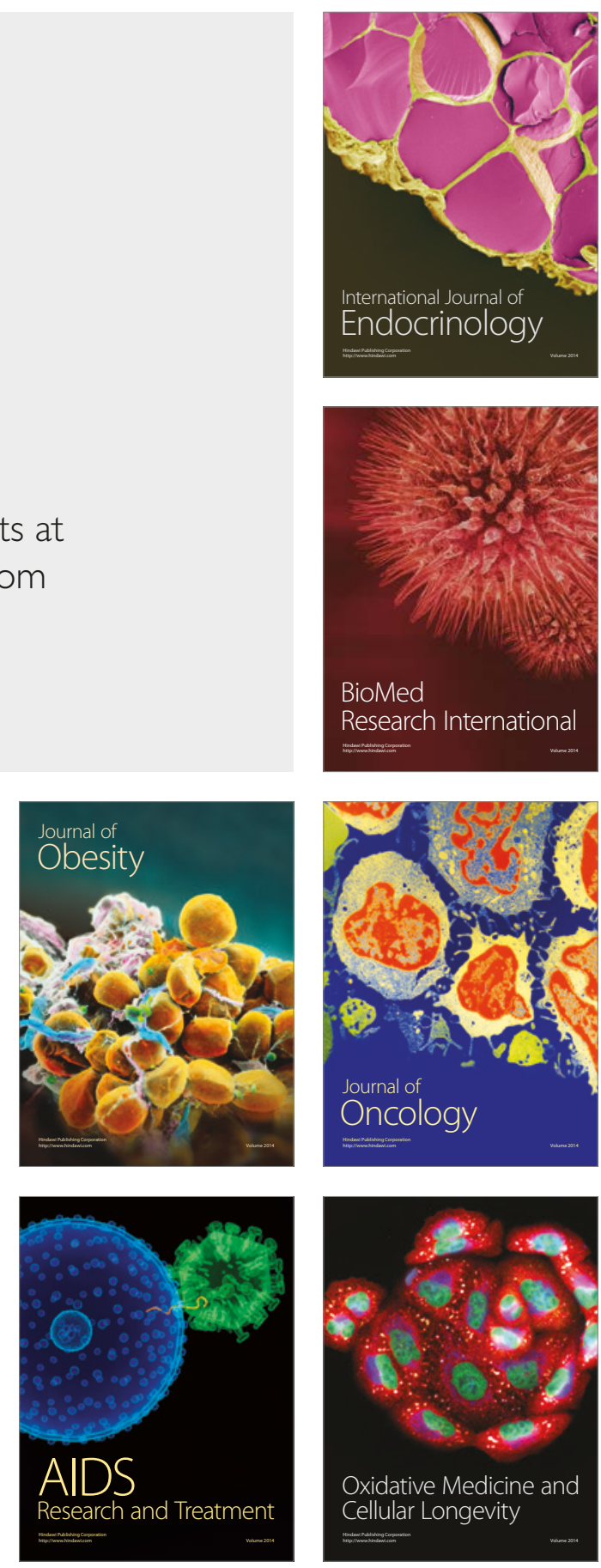